\title{
ЧАСТОТНО-РЕГУЛИРУЕМЫЙ ЭЛЕКТРОПРИВОД ЦЕНТРОБЕЖНЫХ НАСОСНЫХ УСТАНОВОК ДОБЫЧИ НЕФТИ
}

\author{
Чернышева Татьяна Александровна', \\ cherta@tpu.ru \\ Аникин Василий Владимировиче2,3, \\ v-anikin2012@mail.ru \\ Чернышев Игорь Александрович', \\ chia@tpu.ru

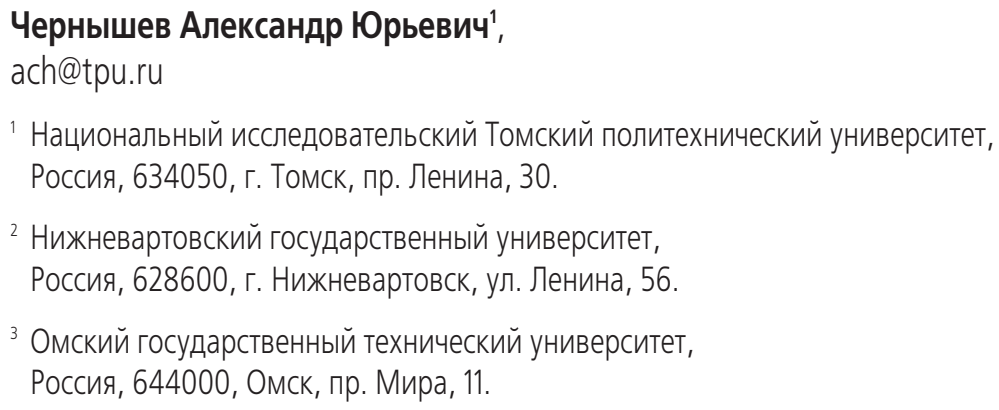

Актуальность работы обусловлена необходимостью снижения аварийности частотно-регулируемых асинхронных электроприводов центробежных насосных установок добычи нефти вследствие перегрева асинхронных двигателей.

Цель: исследовать частотно-регулируемые электроприводы центробежных насосных установок добычи нефти, работающих при скорости выше номинальной.

Методы исследования основаны на использовании теории автоматического управления и имитационного моделирования в программной среде MatLab-Simulink.

Результаты. Составлена схема имитационного моделирования скалярного частотно-регулируемого асинхронного электропривода центробежных насосных установок добычи нефти, учитывающая основные особенности реального электропривода. Рассчитаны механические характеристики электропривода насосной установки. Определены возможные диапазоны регулирования скорости электропривода насосной установки исходя из допустимых потерь мощности в установившихся и переходных режимах.

Выводы. Несмотря на то, что статическая механическая характеристика электропривода с частотой напряжения преобразователя 30 Гц является минимально возможной для добычи нефти с помощью погружных насосов, диапазон регулирования скорости в асинхронном электроприводе погружного насоса необходимо расширить до $D=1: 10$ для плавного пуска электропривода. Установлено, что для уменьшения ударных моментов при пуске асинхронного частотно регулируемого электропривода темп роста частоты на выходе задатчика интенсивности должен составлять несколько десятков секунд даже при пуске на минимальную рабочую скорость электропривода. Неизбежное завышение по мощности асинхронного двигателя для погружного центробежного насоса при его выборе как ближайшего большего из ряда номинальных значений позволяет электроприводу насоса с частотным регулированием скорости работать без перегрева на статических характеристиках выше номинальной. Установлено, что в частотно-регулируемом асинхронном электроприводе центробежного насоса возможное повышение скорости на 10-12 \% от номинальной позволяет увеличить объем добычи нефти без замены оборудования.

\section{Ключевые слова:}

Центробежная насосная установка, асинхронный двигатель,

двухзвенный преобразователь частоты, инвертор напряжения, скалярное регулирование, потери энергии.

\section{Введение}

Бурение скважин на нефть производят после геофизических исследований на предполагаемых участках земной поверхности. После того как скважина пробурена и вскрыт продуктивный пласт, нефть необходимо поднять на поверхность. В начале эксплуатации скважины возможен подъем нефти за счет пластовой природной энергии. При эксплуатации скважины давление в нефтяном коллекторе снижается, и подъем нефти не может осуществляться за счет внутрипластового давле- ния. Добычу нефти можно поддерживать, если в нефтеносный пласт закачивать попутный газ, воздух [1] или жидкость [2] с поверхности. Однако такой способ добычи - газлифтный - находит экономическое обоснование только на ранних стадиях освоения месторождения.

В дальнейшем нефть качают из скважины с помощью штанговых скважинных насосов или погружных электроцентробежных насосов (ЭЦН). Добычу нефти с помощью штанговых скважинных насосов экономически целесообразно осущест- 
влять с глубин от нескольких десятков до сотен метров, предельная глубина опускания штанговых насосных установок доходит до 1500 м.

При отмеченной, сравнительно небольшой глубине залегания нефтеносных пластов станки качалки отличаются большой надежностью и могут работать без капитального ремонта несколько лет. Применяются штанговые насосы в скважинах с низким дебитом. Их производительность находится в диапазоне $5-50 \mathrm{~m}^{3} /$ сут., а подача пластовой жидкости в среднем по Российской Федерации составляет 14,1 т/сут.

Механические станки качалки требуют для своей установки массивные фундаменты, ненадежны при работе в наклонных и горизонтальных скважинах даже на небольшой глубине, так как возникает опасность разрушения обсадной колонны или штока качалки при их взаимном трении. Штанговые насосные установки до недавнего времени оснащались преимущественно нерегулируемыми электроприводами с асинхронными короткозамкнутыми двигателями (АД).

Увеличение межремонтного периода можно добиться, применяя вместо штанговых насосов электроцентробежные насосы, вращающиеся погружными асинхронными двигателями с частотным регулированием скорости. ЭЦН позволяют длительное время откачивать из скважины пластовую жидкость, содержащую песок, газ и коррозионно активные элементы.

Применение частотно-регулируемого асинхронного электропривода позволит уже в ближайшее время если не полностью отказаться от станков качалок, то значительно сократить их количество.

В России [3], особенно в Западной Сибири, уже в настоящее время около $80 \%$ нефти добывается электроцентробежными насосами. Их производительность составляет 114 т/сут., т. е. превышает в 8 раз производительность установки со штанговыми насосами. Очевидно, что ЭЦН применяются в высокодебитных скважинах.

Температура пластовой жидкости на глубине установки электроцентробежного насоса может достигать $90{ }^{\circ} \mathrm{C}$. Гидростатическое давление в зоне установки электродвигателя на глубине $l$ можно найти из выражения

$$
p_{\text {гс }}=\rho_{\text {п.ж }} l,
$$

где $\rho_{\text {п.ж }}-$ плотность пластовой жидкости, кг $/ \mathrm{m}^{3} ; l-$ глубина зоны установки электродвигателя по вертикали, м.

Плотность пластовой жидкости находится в диапазоне от $700 \mathrm{\kappa г} / \mathrm{m}^{3}$ (нефть с незначительными примесями) до максимального значения $1400 \mathrm{\kappa г} / \mathrm{m}^{3}$. Из (1) следует, что на глубине 1500 м при плотности пластовой жидкости $\rho_{\text {п.ж }}=1400 \mathrm{\kappa г} / \mathrm{m}^{3}$ давление в 30не установки электродвигателя может достигать 19 МПа.

На рис. 1 приведена скважина с погружным электроцентробежным насосом. Погружной трех- фазный асинхронный двигатель - 3 с короткозамкнутым ротором получает питание по трехжильному бронированному кабелю - 4. Так как диаметр асинхронного двигателя - 3 в скважине ограничен, при больших его установленных мощностях он имеет большую длину и выполняется секционным. Для увеличения надежной работы двигателя он заполняется маслом с высокой диэлектрической прочностью, близким по свойствам с трансформаторным маслом. Для выравнивания давления масла в двигателе и пластовой жидкости служит компенсатор давления - 1. Пластовая жидкость поступает в колонну через прорези - 2 в колонне, в нижней ее части. Изменения давления масла в асинхронном двигателе - 3 вследствие его нагрева или увеличения глубины погружения приводят к тому, что эластичная диафрагма компенсатора - 1 деформируется, выравнивая давление. Это уменьшает вероятность попадания механических примесей в электродвигатель, увеличивая межремонтный период.

Протектор - 5, расположенный между асинхронным двигателем и насосом, отделяет электродвигатель, заполненный маслом, от насоса, заполненного пластовой жидкостью, и при этом передает вращение от двигателя к насосу. Протектор - 5 защищает двигатель от попадания пластовой жидкости в маслонаполненный электродвигатель со стороны насоса и предотвращает утечку масла при передаче вращения от электродвигателя к насосу.

Газосепаратор - 6 используется для снижения количества газа на входе в насос. Газосепаратор удаляет газ в затрубное пространство и исключает образование газовых пробок в насосе, благодаря чему повышается срок его службы, а вместе с тем и всей скважины.

Многоступенчатый центробежный насос - 7 отличается малым диаметром рабочих ступеней и большим их количеством, доходящим до нескольких сотен. Длина насоса может достигать 6 м. Приемные отверстия насоса и фильтр расположены в нижней его части. Верхний и нижний концы вала насоса установлены в подшипниках скольжения. При большой длине насоса возникающие радиальные вибрации гасятся дополнительными радиальными подшипниками, установленными по длине насоса.

В колонне с центробежным насосом установлен обратный клапан - 8. Он удерживает закаченную в насосно-компрессорную трубу - 10 пластовую жидкость при плановых или аварийных остановках ЭЦН для исключения обратного вращения насоса под действием гидростатического напора жидкости, оставшейся в трубе. Сливной клапан - 9 обеспечивает возврат в затрубное пространство пластовой жидкости.

Многоступенчатый центробежный насос -7 со всем вспомогательным оборудованием (рис. 1) опускают в скважину на колонне насосно-компрессорных труб и подвешивают на шайбе - 11 без дополнительного крепления к скважине. 


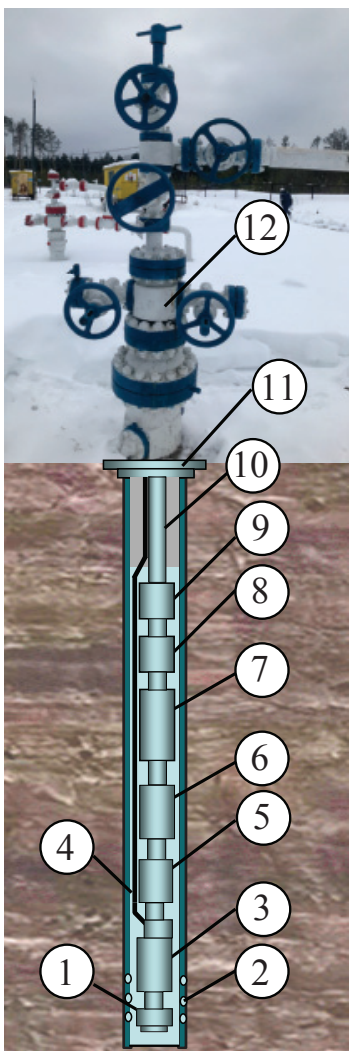

Рис. 1. Основные элементы скважины с электроцентробежныл насосом: 1 - компенсатор давления, 2 - прорези в колон не, 3 - маслозаполненный трехфазный асинхронный дви гатель, 4 - трехжильный бронированный кабель, 5 - про тектор, 6 - газосепаратор, 7 - многоступенчатый центробежный насос, 8 - обратный клапан, 9 - сливной кла пан, 10 - насосно-компрессорная труба, 11 - шайба, 12 устьевое оборудование

Fig. 1. Main elements of the well with electric centrifugal pump: 1 is the pressure compensator, 2 are the slots in the column, 3 is the oil filled three phase induction motor, 4 is the three-core armored cable, 5 is the protector, 6 is the gas separator, 7 is the multistage centrifugal pump, 8 is the back pressure valve, 9 is the overflow valve, 10 is the flow string, 11 is the plate, 12 is the wellhead equipment
Наземное оборудование скважины содержит устьевое оборудование - 12 , предназначенное для сбора и отвода пластовой жидкости и попутного газа, а также электрооборудование - трансформаторы, фильтры, преобразователь частоты системы управления - для плавного пуска и экономичной работы электроцентробежного насоса.

\section{Функциональная схема наземного оборудования} погружного электроцентробежного насоса

Функциональная схема электропривода электрической части наземного оборудования погружного электроцентробежного насоса приведена на рис. 2. Как правило, электрическая энергия к скважинам подводится напряжением 10 или 6 кВ и снижается до стандартного напряжения $400 \mathrm{~B}$ понижающим трансформатором $T V 1$.

Преобразователь частоты (ПЧ) электропривода насоса подключается к понижающему трансформатору TV1 через автомат QF1. Для уменьшения нагрузочных помех в питающей сети из-за широтно-импульсного регулирования напряжения преобразователем частоты на его входе установлен сетевой фильтр (СФ).

Так как для подъема пластовой жидкости погружными электроцентробежными насосами требуется регулирование скорости в небольшом диапазоне $(D=1: 10)$, для вращения насосов вполне достаточно использовать относительно простые общепромышленные преобразователи частоты со скалярным управлением. Причем скалярные электропривода при температурных изменениях параметров двигателя не требуют перестройки регуляторов по причине их отсутствия. На выходе преобразователя частоты устанавливается выходной синусный фильтр (ВСФ). ВСФ преобразует выходное напряжение прямоугольной формы ШИМ-модуляции в напряжение практически синусоидальной формы с коэффициентом искажения, не превышающим 0,05 о.е. Кроме того, ВСФ позволяет исключить волновые электромагнитные и резонансные процессы в длин-

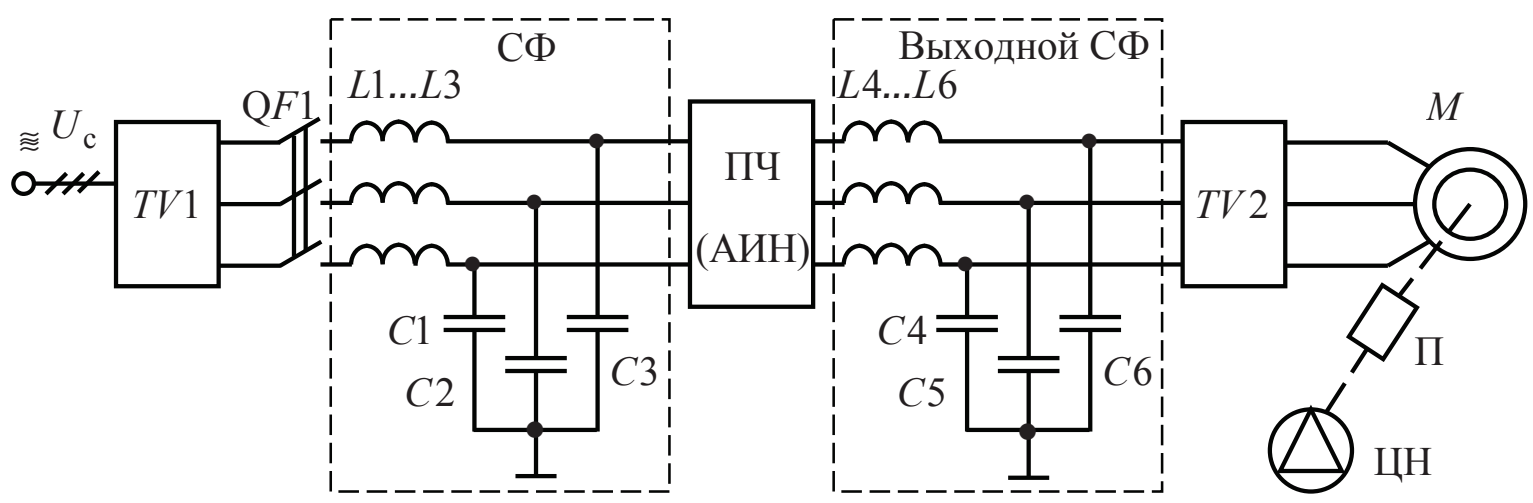

Puc. 2. Функииональная схела электрической части назелного оборудования погружного электроцентробежного насоса: TV1 - пони жающий трансформатор, QF1 - автомат, ПЧ - преобразователь частоты с автономныл инвертором напряжения, TV2 - повышающий трансфорлатор напряжения, $M$ - асинхронный двигатель, П - протектор, ЦН - иентробежный насос

Fig. 2. Functional diagram of the electrical part of the ground equipment of a submersible electric centrifugal pump: TV1 is the step-down transformer, QF1 is the circuit breaker, $\Pi \Psi$ is the frequency converter with autonomous voltage inverter, TV2 is the step-up voltage transformer, $M$ is the induction motor, $\Pi$ is the protector, $Ц H$ is the centrifugal pump 
ном кабеле от преобразователя частоты до погружного асинхронного двигателя в скважине.

Как правило, используемый преобразователь частоты - общепромышленного назначения на линейное напряжение 380 В. А погружные асинхронные двигатели $M$ специального назначения выпускаются на напряжения 600-3000 В. Для согласования напряжений общепромышленного преобразователя частоты и специального погружного асинхронного двигателя применяется повышающий трансформатор TV2. Повышающий трансформатор TV2 и асинхронный двигатель $M$ соединены трехжильным кабелем-удлинителем.

Погружной ЦН подключается к асинхронному двигателю $M$ через протектор (П), и вместе они составляют инерционную массу с моментом инерции $J_{\Sigma}$.

\section{Статические характеристики электропривода} погружного электроцентробежного насоса

Для электропривода любого механизма представляет интерес расчет статических и динамических характеристик для предварительной оценки его работоспособности с различной скоростью необходимого диапазона ее регулирования.

Произведем расчет статических характеристик частотно-регулируемого электропривода и электроцентробежного насоса 1ЭЦН5А-500-800, последний имеет следующие параметры [4]: мощность $N_{\text {нас }}=84,5$ кВт; номинальная частота вращения насоса $n_{\text {нас }}=2820$ об/мин; КПД $\eta_{\text {н }}=0,54$ о.е.; напор $810 \mathrm{~m}$; подача $500 \mathrm{~m}^{3} /$ сут.

Механическую характеристику насоса рассчитаем по формуле:

$$
M_{\text {нас }}=\Delta M_{\text {пост }}+b \omega^{x},
$$

где $x$ - показатель степени; $\Delta M_{\text {пост }}$ - момент постоянных потерь, Н·м; $b$ - коэффициент, определяемый в точке номинальной работы насоса, $\mathrm{H} \cdot \mathrm{M} /\left(\text { рад } \cdot \mathrm{c}^{-1}\right)^{x}$.

$$
\Delta M_{\text {пост }}=a_{\text {н }}\left(\frac{N_{\text {нас }}}{\eta_{\text {н }} \omega_{\text {нас }}}-\frac{N_{\text {нас }}}{\omega_{\text {нас }}}\right) \text {, }
$$

где $\omega_{\text {нас }}-$ номинальная угловая скорость насоса, рад/с; $a_{\text {н }}=\frac{1-\eta_{\mathrm{H}}}{2 \eta_{\mathrm{H}}}-$ коэффициент постоянных потерь насоса в номинальном режиме, о.е.;

Теоретически $[5,6]$ для центробежных насосов показатель степени у скорости в (2) равняется 2. Практически в уравнении механической характеристики центробежного насоса (2) показатель степени у скорости меняется в пределах 2,5-6 для различных конструкций и условий работы насоса [6]. Механическая характеристика центробежного насоса, построенная по (2) с показателем степени $x=2$, изображена на рис. 3 , кривая 1 . Там же приведена механическая характеристика насоса (кривая 2) с показателем степени у скорости $x=3$. Как следует из анализа механических характеристик 1 и 2, при малых скоростях, то есть меньших, чем $\omega \leq 300$ рад/с, они достаточно близки, характери- стики существенно расходятся при больших скоростях $\omega \geq 300$ рад/с. Экспериментальные исследования, проведенные на лабораторном стенде с насосом, позволяют сделать вывод, что показатель степени у скорости ближе к 3 . В дальнейших расчетах будем принимать показатель степени у скорости в механической характеристике насоса $x=3$.

Насос вращается погружным маслонаполненным асинхронным двигателем с короткозамкнутым ротором фирмы АЛНАС типа ЭДТ110-117 M6, имеющим следующие паспортные данные [7]: номинальная мощность $P_{\mathrm{H}}=110 \mathrm{kBт}$; синхронная частота вращения $n_{0}=3000$ об/мин; номинальное линейное напряжение 2300 В; номинальный КПД $\eta_{\text {н }}=0,84$ о.е.; номинальный $\cos \varphi_{\mathrm{H}}=0,84$ о.е.; номинальное скольжение $s_{\text {н }}=0,06$ о.е.; количество секций - 1 ; длина $8,24 \mathrm{~m}$; масса - $571 \mathrm{\kappa г;} \mathrm{скорость} \mathrm{охлаждающей}$ жидкости не менее $0,3 \mathrm{~m} / \mathrm{c}$; отношение максимального момента $M_{\max }$ к номинальному $M_{\text {н }}$ моменту погружного электродвигателя должно быть не менее 2 (ТУ 3381-026-21945400-97), принято $\lambda_{\mathrm{M}}=2,2$ о.е.

Двигатель завышен по мощности, однако окончательный вывод можно сделать только по завершении расчетов статических и динамических характеристик, а также по тепловому режиму работы электропривода.

По каталожным данным двигателя, в соответствии с методикой, изложенной в [8, 9], определены параметры Т-образной схемы замещения асинхронного двигателя ЭДТ110-117M6: $R_{1}=2,294$ Ом - активное сопротивление обмотки статора; $R_{2}^{\prime}=2,253$ Ом - активное сопротивление обмотки ротора, приведенное $\mathrm{k}$ обмотке статора; $X_{1 \sigma н}=3,131$ Ом - индуктивное сопротивление рассеяния обмотки статора при номинальной частоте питающей сети $f_{1 H} ; X_{2 н}^{\prime}=4,247$ Ом - индуктивное сопротивление рассеяния обмотки ротора, приведенное к обмотке статора при номинальной частоте питающей сети $f_{1 \mathrm{H}} ; X_{\mu \mathrm{H}}=120,295 \mathrm{OM}-$ индуктивное сопротивление контура намагничивания при номинальной частоте питающей сети $f_{1 н}$.

Естественная механическая характеристика асинхронного двигателя, построенная по паспортным данным, приведена на рис. 3, ее контрольные точки, отмеченные треугольниками - $\triangle$, полностью совпадают с каталожными данными двигателя ЭДТ110-117 М6. Точка 3 - синхронная скорость, режим идеального холостого хода; 4 - точка номинального режима работы асинхронного двигателя; 5 - максимальный момент асинхронного двигателя.

Искусственные механические характеристики асинхронного частотно-регулируемого электропривода с автономным инвертором напряжения можно рассчитать по уравнению:

$$
M=\frac{3\left(U_{1 j}-2 U_{V D}-2 U_{V T}\right)^{2} R_{2}^{\prime}}{\omega_{0 \mathrm{j}} \mathrm{s}\left[\begin{array}{l}
\left(X_{1 \sigma \mathrm{H}}+X_{1 \text { доб }}+X_{2 \sigma \mathrm{H}}^{\prime}\right)^{2} f_{1 *}^{2}+ \\
+\left(R_{1}+R_{1 \text { доб }}+\frac{R_{2}^{\prime}}{s}\right)^{2}+\left(\frac{\left(R_{1}+R_{1 \text { доб }}\right) R_{2}^{\prime}}{s X_{\mu \mathrm{H}} f_{1 *}}\right)^{2}
\end{array}\right]},
$$


где $R_{1 д о б}$ - добавочное активное сопротивление кабеля-удлинителя, проводов, соединяющих преобразователь частоты и обмотки статора асинхронного двигателя; Ом; $X_{1 \text { доб }}-$ добавочное индуктивное сопротивление кабеля-удлинителя, проводов, соединяющих преобразователь частоты и обмотки статора асинхронного двигателя; Ом; $U_{V D}=1$, В - прямое падение напряжение на диоде выпрямительного моста двухзвенного преобразователя частоты; $U_{V T} \approx 1,5$, В - прямое падение напряжение на IGBTтранзисторе инвертора напряжения; $f_{1^{*}}=f_{1 j} / f_{1 \mathrm{H}}-$ относительная частота напряжения инвертора; $f_{1 j}$ текущее значение частоты напряжения инвертора; $U_{1 j}$ - фазное напряжение обмоток статора асинхронного двигателя; $\omega_{0 \mathrm{j}}=\frac{\pi f_{1 j}}{p}-$ синхронная угловая скорость; $p$ - число пар полюсов асинхронного двигателя. Остальные обозначения физических величин общепринятые для Т-образной схемы замещения асинхронного короткозамкнутого двигателя.

Активное сопротивление одной жилы кабеляудлинителя $R_{1 \text { каб }}$, имеющего наибольшее влияние на добавочное сопротивление $R_{1 \text { доб }}$ можно найти из выражения:

$$
\begin{gathered}
R_{1 \text { каб }}=\frac{l \rho}{S}[1+\alpha(\theta-20)]= \\
=\frac{1 \cdot 10^{3} \cdot 1,79 \cdot 10^{-3}}{16}[1+\alpha(\theta-20)]=0,1428 \mathrm{OM},
\end{gathered}
$$

где $l=1 \cdot 10^{3}$ - длина кабеля, м; $\rho=1,79 \cdot 10^{-3} \mathrm{OM} \cdot \mathrm{MM}^{2} / \mathrm{M}-$ удельное электрическое сопротивление меди при температуре $20^{\circ} \mathrm{C}, \mathrm{Om} \cdot \mathrm{mm}^{2} / \mathrm{m} ; S=16$ - сечение жилы кабеля мм $^{2} ; \alpha=0,00393$ - температурный коэффициент электрического сопротивления меди, $1 /{ }^{\circ} \mathrm{C}[10]$.

Индуктивное сопротивление одного километра жилы кабеля удлинителя находится по формуле [10]:

$$
\begin{gathered}
X_{0}=\left(0,145 \lg \frac{2 D_{\mathrm{cp}}}{d}+0,0157 \mu_{r}\right) \cdot 10^{-3}= \\
=\left(0,145 \lg \frac{2 \cdot 7,3}{4,5}+0,0157 \cdot 1\right) \cdot 10^{-3}=8,96 \cdot 10^{-5} \frac{\mathrm{OM}}{\mathrm{M}},
\end{gathered}
$$

где $D_{\text {ср }}=7,3$ - расстояние между центрами жил кабелей, мм; $d=4,5$ - диаметр токоведущей жилы кабеля, мм; $\mu_{r}=1$ - магнитная проницаемость меди.

Тогда

$$
X_{1 \text { каб }}=X_{0} l=6,67 \cdot 10^{-5} \cdot 1 \cdot 10^{3}=0,0667 \text { Ом. }
$$

Известно [11-16], что при работе преобразователя частоты с инвертором напряжения в режиме ШИМ-модуляции выходное напряжение преобразователя уменьшается в 1,174 раза со стандартного фазного $U_{1 \phi н}=220$ В до $U_{1 \phi н}=181,37 \mathrm{~B}$ за счет деления между обмотками асинхронного двигателя напряжения звена постоянного тока на две равные части. Поднять напряжение инвертора с ШИМ-модуляцией можно введением третьей гармоники в сигнал управления инвертором [16]. Максимальное выходное напряжение инвертора в этом случае достигает $U_{1 \phi 1}=209,3 \mathrm{~B}$, причем третья гармоника напряжения в выходном сигнале инвертора момент асинхронного двигателя не создает.

При частотах преобразователя меньших номинальной влияние активного сопротивления статорной цепи $R_{1 \text { доб }}$ Уменьшает критический момент асинхронного двигателя. Его увеличение можно осуществить, изменив вольт-частотную характеристику преобразователя частоты.

И, наконец, в рассматриваемой схеме электропривода (рис. 2), когда асинхронный двигатель удален от преобразователя частоты на расстояние в несколько сотен метров по условиям работы в скважине, напряжение на двигателе можно поднять, используя отпайки повышающего трансформатора напряжения TV2 на выходе преобразователя частоты (рис. 2). Рассмотренные три фактора позволяют получить механические характеристики электропривода со скалярным управлением и законом управления инвертором напряжения $U_{1 j} / f_{1 j}^{2}=$ const, приведенные на рис. 3 .

Как следует из рекомендаций фирмы-изготовителя погружного асинхронного двигателя ЭДТ110-117 M6, скорость охлаждающей пластовой жидкости между стенкой обсадной трубы скважины и внешним кожухом асинхронного двигателя при работе центробежного насоса не должна быть меньше $0,3 \mathrm{~m} / \mathrm{c}$. Такая скорость охлаждающей пластовой жидкости достигается при питании двигателя центробежного насоса напряжением с частотой не ниже 30 Гц. Кроме того, частота 30 Гц питания асинхронного двигателя позволяет обеспечить минимальные условия смазывания подшипников погружного асинхронного двигателя насоса и устранить риск «полусухого трения», что является одной из причин выхода асинхронного двигателя погружного насоса из строя. Таким образом, статическая характеристика электропривода с частотой напряжения преобразователя 30 Гц является минимально возможной для добычи нефти с помощью погружных насосов.

Однако нижний предел диапазона регулирования асинхронного электропривода насоса необходимо расширить для плавного пуска электропривода от задатчика интенсивности, изменив вольтчастотную характеристику преобразователя частоты со скалярным законом регулирования (механическая характеристика при 20 Гц на рис. 3).

На рис. 3 приведены механические характеристики электропривода для работы во II зоне - в диапазоне скоростей выше естественной характеристики двигателя. Регулирование скорости во второй зоне происходит с законом регулирования $f_{1 j}=\mathrm{var}$; $U_{1 \phi}=U_{1 \mathrm{H}}$. Регулирование в зоне выше естественной характеристики происходит с ослаблением потока и с постоянной мощностью. Механическая характеристика механизма, работающего с постоянной мощностью, приведена на рис. 3 , кривая 6 . Она расходится с механической характеристикой насоса - 
нелинейно-возрастающей механической характеристикой (кривая 2). Однако если потери в асинхронном двигателе с погружным центробежным насосом в скважине на характеристиках во второй зоне не превысят номинальных потерь в двигателе, велик соблазн работать со скоростью выше номинальной с целью увеличения производительности скважины и объема добычи нефти.

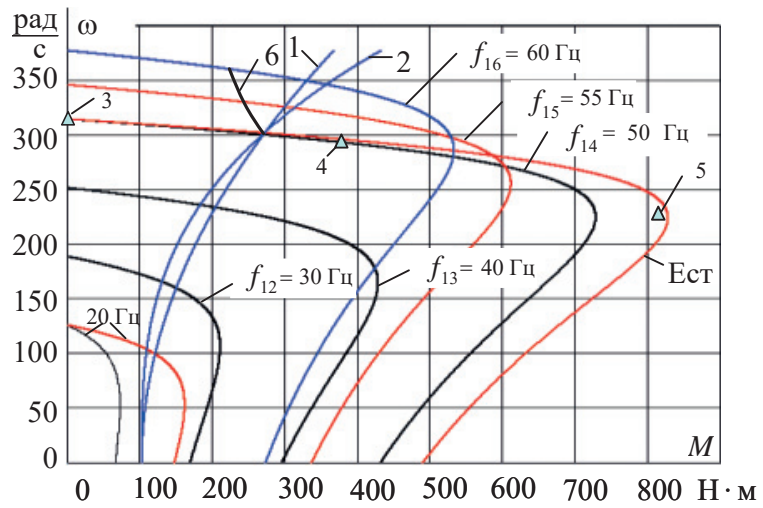

Pис.3. Механические характеристики скалярного асинхронного электропривода погружного иентробежного насоса: 1 механическая характеристика нагрузки с показателем скорости $x=2 ; 2$ - механическая характеристика нагруз ки (иентробежного насоса) с показателем скорости $x=3$, 3 - синхронная скорость, режим идеального холостого хо да, 4 - точка номинального режима работы асинхронно 20 двигателя, 5 - максимальный молент асинхронного двигателя; 6 - механическая характеристика механиз ма, работающего с постоянной мощностью

Fig. 3. Mechanical characteristics of a scalar controlled asynchro nous electric drive of submersible centrifugal pump: 1 is the mechanical load characteristic with speed indicator $x=2 ; 2$ is the mechanical characteristic of the load (centrifugal pump) with a speed indicator $x=3 ; 3$ is the synchronous speed in ide al no-load mode; 4 is the point of rated power operation mode of induction mode; 5 is the maximum torque of induction mo tor; 6 is the mechanical characteristic of constant power mechanism

\section{Моделирование работы электропривода центробежного насоса в динамике}

Провести проверку электропривода центробежных насосных установок добычи нефти по тепловому режиму работы теоретически достаточно сложно, так как отсутствует постоянная времени нагрева двигателя и нет обоснованных методик для ее определения. Однако несложно найти потери в двигателе практически во всех его режимах работы, моделируя асинхронный электропривод в программной среде MatLab-Simulink.

На рис. 4 приведены графики переходных процессов задания частоты с выхода $S$-образного задатчика интенсивности в функции времени $f_{\text {зад }}=f(t)$ и графики переходных процессов момента асинхронного двигателя $M=f(t)$ и его скорости $\omega=f(t)$ при пуске на три фиксированные скорости, соответствующие частотам задания: 30,50 и 60 Гц.

Исследования переходных процессов в асинхронном электроприводе со скалярным регулиро- ванием скорости проводились с учетом следующих особенностей электропривода:

- ШИМ-модуляции выходного напряжения инвертора;

- наличия третьей гармоники в кривой предмодуляции напряжения управления инвертором напряжения двухзвенного преобразователя частоты;

- наличия активных и индуктивных сопротивлений проводов инвертора напряжения, кабельных линий на входе и выходе инвертора напряжения;

- падения напряжения в диодах выпрямительного моста и IJBT-транзисторах инвертора;

- нелинейной вольт-частотной характеристики инвертора, аппроксимируемой тремя отрезками прямых линий;

- наличия задержанной отрицательной обратной связи по току двигателя или токовой отсечки.

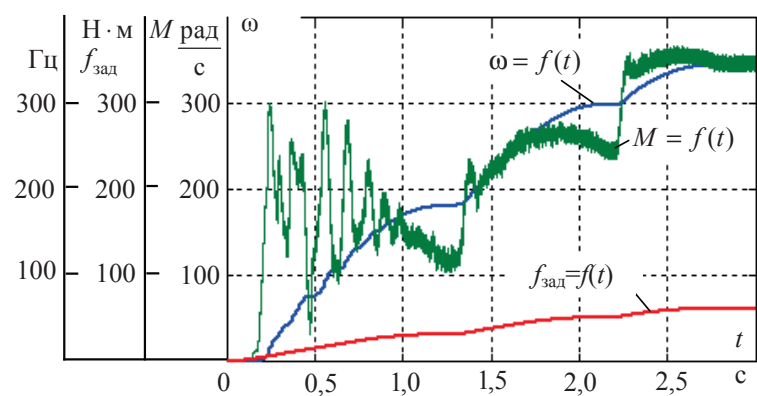

Рис.4. Графики переходных проиессов задания частоты $f_{\text {зад }}=f(t)$, молента асинхронного двигателя ЭДТ110-117 M6 $M=f(t)$, его скорости $\omega=f(t)$ при пуске

Fig. 4. Transients of frequency reference $f_{\text {зад }}=f(t)$, torque of induction motor EDT110-117 M6 M=f(t), its speed $\omega=f(t)$ for starting

Для наглядности ШИМ-модуляции инвертора напряжения в схеме имитационной модели электропривода на выходе преобразователя частоты отсутствует выходной синусный фильтр (ВСФ), что не сказывается на достоверности отображения основных процессов в электроприводе, так как асинхронный электродвигатель - инерционное звено второго порядка и сам способен сглаживать колебания скорости в переходных режимах.

Динамические броски момента АД (рис. 4) можно уменьшить, увеличив время пуска электропривода задатчиком интенсивности или применяя для электропривода центробежного насоса системы скалярного управления с отрицательной обратной связью по скорости или системы векторного управления без датчика скорости, а скорость определять с помощью наблюдателей [17-22]. Однако применение последних способов регулирования для улучшения качества переходных процессов момента АД возможно только для ограниченного числа скважинных электроприводов.

Для электропривода центробежного насоса, выполненного в соответствии с функциональной схемой, приведенной на рис. 2, потребляемую из сети 
мощность $P_{s}$ можно найти через мгновенные значения фазных токов и напряжений:

$$
P_{s}=i_{1 A} u_{1 A}+i_{1 B} u_{1 B}+i_{1 C} u_{1 C},
$$

где $i_{1 A}, i_{1 B}, i_{1 C}$ - мгновенные значения фазных токов асинхронного электропривода; $u_{1 A}, u_{1 B}, u_{1 C}$ - мгновенные значения фазных напряжений электропривода.

Мгновенные значения мощности на валу асинхронного двигателя найдем из выражения

$$
P_{m}=M \omega,
$$

где $M$ - мгновенное значение момента асинхронного двигателя, найденное по результатам расчета переходных процессов, $\mathrm{H} \cdot \mathrm{m} ; \omega$ - мгновенное значение скорости асинхронного двигателя, рад/с.

Потери мощности в асинхронном двигателе находятся как разность потребляемой мощности электроприводом из сети и мощности на валу асинхронного двигателя:

$$
\Delta P=P_{s}-P_{m} .
$$

Суперблок (Subsystem 3) расчета потерь мощности в асинхронном электроприводе, выполненный в программной среде MatLab-Simulink, вычисляющий мощности по выражениям (3)-(5), приведен на рис. 5. На выходе суперблока установлен осциллограф (Scop 8) для визуального контроля переходных процессов мощности и цифровые индикаторы Loss P1-Loss P3 для точного измерения установившихся значений мощности.

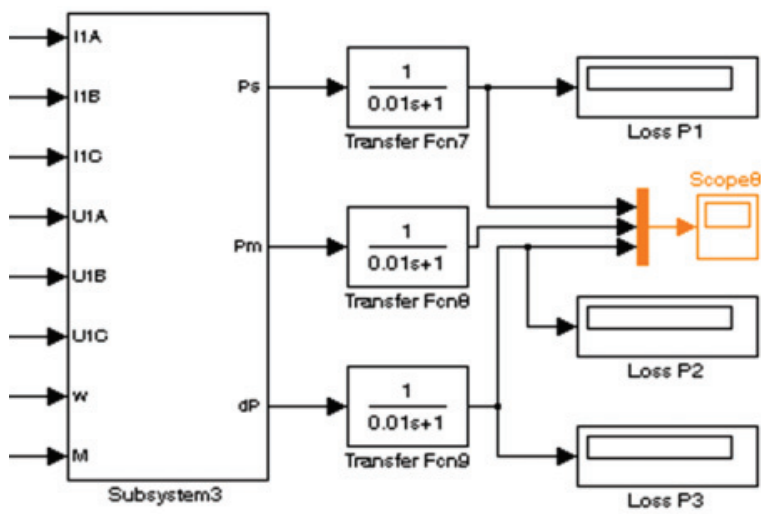

Pис.5. Суперблок расчета потерь мощности в асинхронном электроприводе

Fig. 5. Superblock for calculating power losses in asynchronous electric drive

Результаты расчета переходных процессов потребляемой из сети мощности асинхронным электроприводом $P_{s}$, мощности на валу асинхронного двигателя $P_{m}$ и мощности потерь $\Delta P$ приведены на рис 6.

Анализ графиков рис. 6 показывает, что на начальном участке переходных процессов $0-0,1$ с в частотно-регулируемом электроприводе возникают большие потери мощности вследствие заряда конденсатора емкостного фильтра преобразователя частоты. По окончании заряда конденсатора фильтра возможен процесс пуска электропривода.
По мере разгона асинхронного двигателя потери мощности $\Delta P$ на фиксированных скоростях увеличиваются.

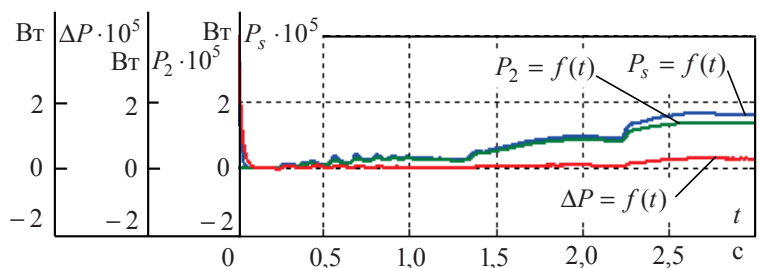

Pис.6. Переходные процессы потребляемой асинхронныл әлектроприводол мошности из сети $P_{\mathrm{s}}$, мошности на валу асинхронного двигателя $P_{\mathrm{m}}$ и потерь в асинхронном двигателе $\triangle P$ при разгоне и работе двигателя на трех фиксированных скоростях

Fig. 6. Transients of power $P_{\mathrm{s}}$ consumed by asynchronous electric drive from the power supply, mechanical power $P_{\mathrm{m}}$ on the shaft of induction motor and power losses $\Delta P$ in induction motor for its starting and operation with three fixed speed $v a$ lues

Численные значения потребляемой электроприводом мощности $P_{s}$, мощности на валу асинхронного двигателя $P_{m}$, потери в двигателе $\Delta P$ сведены в таблицу. Там же приведены значения коэффициента полезного действия асинхронного двигателя для каждой из фиксированных частот преобразователя частоты.

таблица. Значения потребляемой электроприводом мощности, мощности на валу асинхронного двигателя, потери в двигателе

Table. $\quad$ Values of power consumed by the electric drive, power on the shaft of the induction motor, power losses in the motor

\begin{tabular}{|c|c|c|c|c|c|}
\hline$f_{1 i}$, Гц $(\mathrm{Hz})$ & 30 & 50 & 55 & 57 & 60 \\
\hline$P_{s}$ кВт $(\mathrm{kW})$ & 26,71 & 93,19 & 122,5 & 137,8 & 161,3 \\
\hline$P_{m}$ & 25,31 & 84,86 & 107,5 & 118,1 & 133,5 \\
\hline$\Delta P$ & 1,4 & 8,33 & 15,0 & 19,7 & 27,8 \\
\hline$\eta$, o.e. (r.u.) & 0,947 & 0,91 & 0,878 & 0,857 & 0,62 \\
\hline
\end{tabular}

Номинальные потери мощности асинхронного двигателя можно найти по паспортным данным из выражения $\Delta P_{\mathrm{H}}=P_{\mathrm{H}}\left(1-\eta_{\mathrm{H}}\right) / \eta_{\text {н }}$, для двигателя фирмы АЛНАС типа ЭДТ110-117 М6 они равны $\Delta P_{\mathrm{H}}=20,95 \kappa \mathrm{\kappa T}$.

Анализ мощностей, полученных по результатам математического моделирования в программной среде MatLab-Simulink и номинальных потерь $\Delta P_{\text {н }}$ в асинхронном двигателе типа ЭДТ110-117 M6, показывает, что рост производительности центробежных насосных установок добычи нефти при частотном регулировании скорости приводного асинхронного двигателя вверх от номинальной скорости увеличением частоты возможно на 10-12\%, но только в электроприводах с завышенной мощностью установленных двигателя и преобразователя частоты. В практических расчетах выбора электродвигателя по мощности в большинстве случаев так и поступают - для механизма выбирают двигатель ближайшей большей мощности из стандартного ряда значений. 


\section{Выводы}

1. Несмотря на то, что статическая механическая характеристика электропривода с частотой напряжения преобразователя 30 Гц является минимально возможной для добычи нефти с помощью погружных насосов, диапазон регулирования скорости в асинхронном электроприводе погружного насоса необходимо расширить до $\mathrm{D}=1: 10$ для плавного пуска электропривода.

2. Установлено, что для уменьшения ударных моментов при пуске асинхронного частотно регулируемого электропривода темп роста частоты на выходе задатчика интенсивности должен быть увеличен до нескольких десятков секунд даже при пуске на минимальную рабочую скорость электропривода.

\section{СПИСОК ЛИТЕРАТУРЫ}

1. Лабораторное моделирование процесса закачки воздуха высокого давления на месторождениях баженовской свиты / Т.М. Бондаренко, Е.Ю. Попов, А.Н. Черемисин, Е.В. Козлова, И.А. Карпов, Н.В. Морозов // Нефтяное хозяйство. - 2017. № 3. - C. 34-39.

2. Планирование, проведение и использование результатов индикаторных исследований для контроля разработки нефтяного месторождения / Б.Г. Ганиев, П.Н. Кубарев, А.Г. Камышников, К.В. Шишкин // Нефтяное хозяйство. - 2017. - № 6. C. $44-47$.

3. Исследование характеристик электроцентробежного насоса с эжектором на входе при откачке газожикостных смесей / В.С. Вербицкий, К.А. Горидько, А.Э. Федоров, А.Н. Дроздов // Нефтяное хозяйство. - 2016. - № 9. - С. 106-109.

4. Установки погружных центробежных насосов типа УЭцН для добычи нефти. Каталог / С.З. Кузьминов, Ю.И. Стариков, В.0. Сухомлинов, В.Н. Филиппов, Р.И. Чуева. - М.: ЦИНТИхимнефтемаш, 1987. - $216 \mathrm{c.}$

5. Ковалев А.Ю., Кузнецов Е.М., Аникин В.В. Электротехнологические установки для нефтедобычи. - Омск: Минобрнауки России, 2015. - 160 с.

6. Соколов М.М. Автоматизированный электропривод общепромышленных механизмов. - М.: Энергия, 1976. - 488 с.

7. Погружные электродвигатели (ПЭД). URL: http://www.rimera.com/upload/catalog/progruzhnyc-elektrodvig-ped.pdf.com (дата обращения 04.09.2019).

8. Чернышев А.Ю., Дементьев Ю.Н., Чернышев И.А. Электропривод переменного тока. - М.: Изд-во «Юрайт», 2019. - 215 с.

9. Боловин Е.В., Глазырин А.С. Методы идентификации параметров погружных асинхронных электродвигателей электроприводных центробежных насосов для добычи нефти // Известия Томского политехнического университета. Инжиниринг георесурсов. - 2017. - Т. 328. - № 1. - С. 123-131.

10. Бобылев А.В. Механические и технологические свойства металлов. Справочник. - М.: Металлургия, 1984. - 208 с.

11. Шрейнер Р.Т. Математическое моделирование электроприводов переменного тока с полупроводниковыми преобразователями частоты. - Екатеринбург: УРО РАН, 2000. - 654 с.

12. Idir A., Kidouche M. Real-time simulation of V/F scalar controlled induction motor using RT-Lab platform for educational purpose // Proc. of the 2013 International Conference on Systems, Control and Informatics. - Venice, Italy, September 28-30, 2013. - P. 189-192.
3. Установлено, что неизбежное завышение по мощности асинхронного двигателя для погружного центробежного насоса при его выборе как ближайшего большего из ряда номинальных значений позволяет электроприводу насоса с частотным регулированием скорости работать без перегрева на статических характеристиках выше номинальной.

4. Установлено, что в частотно-регулируемых асинхронных электроприводах увеличение скорости электропривода насоса, как правило, не превышает 10-12 \% от номинальной, что позволяет увеличить объем добычи нефти без замены оборудования.

Исследование проводится в Томском политехническом университете в рамках гранта Програмлы повышения конкурентоспособности Томского политехнического университета.

13. Bilal Akin, Nishant Garg. Scalar (V/f) control of 3-phase induction motors. - Dallas, Texas: Texas Instruments, Inc. Systems and Applications, 2000. - $27 \mathrm{p}$.

14. Akroum H., Kidouche M., Aibeche A. Scalar control of induction motor drives Using dSPACE DS1104 // Proc. of the 2013 International Conference on Systems, Control and Informatics. - Venice, 2013. - P. 322-327.

15. Real-time modeling and simulation of an active power filter / S. Beaulieu, M. Ouhrouche, C. Dufour, P.F. Allaire // Proc. of Ninth IASTED International Conference on Power and Energy Systems - PES 2007. - Clearwater, Florida, USA, January 3-5, 2007. - P. 128-133.

16. Удут Л.С., Чернышев А.Ю., Гусев Н.В. Разработка и моделирование асинхронных электроприводов со скалярным управлением // Изв. вузов. Электромеханика. - 2015. № 6 (359). - C. 43-49.

17. Разработка наблюдателя полного порядка с оперативным мониторингом момента сопротивления для погружных асинхронных двигателей / А.С. Глазырин, С.Н. Кладиев, К.С. Афанасьев, В.В. Тимошкин, В.И. Полищук, С. Халас // Известия Томского политехнического университета. Инжиниринг георесурсов. - 2018. - Т. 329. - № 2. - С. 118-126.

18. Herman I., Vaclavek P. Load torque and moment of inertia observability analysis for alternating current drive sensorless control // IECON Proceedings, Industrial Electronics Conference. - Montreal, Canada, 2012. - P. 1864-1869.

19. Binder B.J.T., Pavlov A., Johansen T.A. Estimation of flow rate and viscosity in a well with an electric submersible pump using moving horizon estimation // IFAC-Papers on line. - 2015. V. 28 (6). - P. 140-146.

20. Rabbi S.F., Constantine M., Rahman M.A. A novel sensorless IPM motor drive for electric submersible pumps // IEEE International Electric Machines and Drives Conference. - Miami, USA, 2017. - Conference Paper № 8002199. - P. 1-8.

21. Messaoudi M., Sbita L. Sensorless direct torque and flux control of induction motor based on MRAS and Luenberger observer // International Conference on Green Energy and Conversion Systems. - Hammamet, Tunisia, 2017. - Conference Paper № 8066224. - P. 1-7.

22. Online robust estimation of flux and load torque in induction motors / M. Bahloul, L. Chrifi-Alaoui, A.N. Vargas, M. Chaabane, S. Drid // International Journal of Advanced Manufacturing Technology. - 2017. - V. 94 (5-8). - P. 2703-2713. 


\section{Информация об авторах}

Чернышева T.A., старший преподаватель отделения общетехнических дисциплин Школы базовой инженерной подготовки Национального исследовательского Томского политехнического университета.

Аникин B.B., старший преподаватель кафедры автоматизации и робототехники Нижневартовского государственного университета, Россия, 628600, г. Нижневартовск, ул. Ленина, 56; соискатель кафедры электрической техники Омского государственного технического университета.

Чернышев И.А., кандидат технических наук, доцент отделения электроэнергетики и электротехники Инженерной школы энергетики Национального исследовательского Томского политехнического университета.

Чернышев А.Ю., кандидат технических наук, доцент отделения электроэнергетики и электротехники Инженерной школы энергетики Национального исследовательского Томского политехнического университета. 
UDC 622-83-52:621.678:622.276.5

\title{
VARIABLE SPEED ELECTRIC DRIVE OF CENTRIFUGAL PUMP IN OIL LIFTING PLANTS
}

Tatyana A. Chernysheva',

cherta@tpu.ru

\author{
Vasiliy V. Anikin ${ }^{2,3}$, \\ v-anikin2012@mail.ru
}

Igor A. Chernyshev', chia@tpu.ru

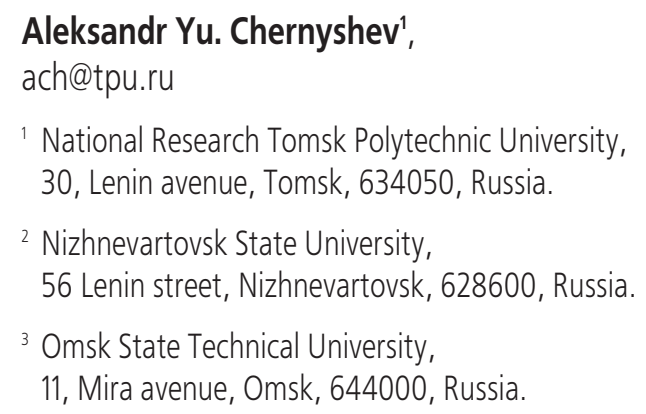

Relevance of the work is caused by the need to create reliable frequency-controlled variable speed asynchronous induction motor drives of centrifugal pumping units for oil lifting.

The main aim of the research is to study the frequency-controlled electric drives of centrifugal pumping units for oil lifting that operate at a speed higher than the nominal (rated) one.

Methods of the research are based on the use of the automatic control theory and simulation in the MatLab-Simulink software environment.

Results. The authors have made up the block scheme for simulation of a scalar frequency-controlled asynchronous induction motor drive of centrifugal pumping units for oil lifting. The scheme takes into account the main features of the real electric drive. The authors calculated mechanical characteristics of the electric drive of the pumping unit and determined the accessible ranges for controlling the speed of the electric drive of the pump unit based on the allowable power losses in steady-state and transient modes.

Findings. Despite the fact that the static mechanical characteristic of an electric drive with ac converter frequency of $30 \mathrm{~Hz}$ is the minimum possible for oil lifting using submersible pumps, the range of speed control in an asynchronous induction motor drive of a submersible pump must be expanded to $D=1: 10$ for a soft starting of the electric drive. It was found that in order to reduce high torque when starting an asynchronous frequency-controlled induction motor drive the frequency ramp-up rate at the output of the ramp generator should be several tens of seconds even for starting at the minimum operating speed of the electric drive. It was established that the inevitable power overrating of the induction motor for a submersible centrifugal pump, when it is selected as the closest higher one from a series of nominal values, allows the electric drive of the pump with frequency speed control to work without overheating on static characteristics above the nominal (rated) one. It was found that in frequency-controlled asynchronous induction motor drives the increase in the pump electric drive speed as a rule does not exceed 10-12\% of the nominal (rated) one, which allows increasing oil lifting output without upgrading the electric drive equipment.

\section{Key words:}

Centrifugal pumping unit, induction motor, indirect ac power frequency converter, voltage inverter, scalar control, energy loss.

The research is carried out at Tomsk Polytechnic University within the grant of the Program of Tomsk Polytechnic University Competitiveness Enhancement.

\section{REFERENCES}

1. Bondarenko T.M., Popov E.Yu., Cheremisin A.N., Kozlova E.V., Karpov I.A., Morozov N.V. Laboratory modeling of high-pressure air injection in oil fields of Bazhenov formation. Oil industry, 2017, no. 3 P. 34-39. In Rus.

2. Ganiev B.G., Kubarev P.N., Kamyshnikov A.G., Shishkin K.V. Tracer surveys as a reservoir management tool. Oil industry, 2017, no. 6, P. 44- 47. In Rus.

3. Verbitsky V.S., Goridko K.A., Fedorov A.E. Drozdov A.N. Experimental studies of electric submersible pump performance with ejector at pump inlet when liquid-gas mixture delivering. Oil industry, 2016, no. 9, P. 106-109. In Rus.

4. Kuzminov S.Z., Starikov Yu.I., Sukhomlinov V.0., Filippov V.N., Chueva R.I. Ustanovki pogruzhnykh tsentrobezhnykh nasosov tipa UETSN dlya dobychi nefti. Katalog [Submersible centrifugal pumps of ESCP type for oil lifting. Catalogue]. Moscow, Central Institute of Scientific and Technical Information of Chemical and Petroleum Engineering Publ., 1987. 216 p.

5. Kovalev A.Yu., Kuznetsov E.M., Anikin V.V. Elektrotekhnologicheskie ustanovki dlya neftedobychi [Electrotechnological installations for oil lifting]. Omsk, Ministry of Education and Science of the Russian Federation Publ., 2015. 160 p.

6. Sokolov M.M. Avtomatizirovanny elektroprivod obshchepromyshlennykh mekhanizmov [Automated electric drive of general industrial mechanisms]. Moscow, Energiya Publ., 1976. 488 p.

7. Pogruzhnye elektrodvigateli [Submersible motors]. Available at: http://www.rimera.com/upload/catalog/progruzhnyc-elektrodvig-ped.pdf.com (accessed 4 September 2019). 
8. Chernyshev A.Yu., Dementev Yu.N., Chernyshev I.A. Elektroprivod perevennogo toka [AC electric drive]. Moscow, Yurait Publ., 2019. $215 \mathrm{p}$.

9. Bolovin E.V., Glazyrin A.S. Method for identifying parameters of submersible induction motors of electrical submersible pump units for oil production. Bulletin of the Tomsk Polytechnic University. Geo Assets Engineering, 2017, vol. 328, no. 1, P. 123-131. In Rus.

10. Bobylev A.V. Mekhanicheskie i tekhnologicheskie svoystua metal lov [Mechanical and technological properties of metals]. Moscow: Metalurgiya Publ., 1984. 208 p.

11. Shreyner R.T. Matematicheskoe modelirovanie elektroprivodov peremennogo toka s poluprovodnikovymi preobrazovatelyami chastoty [Mathematical modeling of AC drives with semiconductor frequency converters]. Ekaterinburg: Ural Branch of the Russian Academy of Sciences Publ., 2000. 654 p.

12. Idir A., Kidouche M. Real-time simulation of V/F scalar controlled induction motor using RT-Lab platform for educational purpose. Proc. of the 2013 International Conference on Systems, Control and Informatics. Venice, Italy, September 28-30, 2013. pp. 189-192.

13. Bilal Akin, Nishant Garg. Scalar (V/f) control of 3-phase induction motors. Dallas, Texas, Texas Instruments, Inc. Systems and Applications, 2000.27 p.

14. Akroum H., Kidouche M., Aibeche A. Scalar control of induction motor drives Using dSPACE DS1104. Proc. of the 2013 Interna tional Conference on Systems, Control and Informatics. Venice, 2013. pp. 322-327.

15. Beaulieu S., Ouhrouche M., Dufour C., Allaire P.F. Real-time modeling and simulation of an active power filter. Proc. of Ninth IASTED International Conference on Power and Energy Systems PES 2007. Clearwater, Florida, USA, January 3-5, 2007. pp. 128-133.
16. Beaulieu S., Ouhrouche M., Dufour C., Allaire P.F. Real-time modeling and simulation of an active power filter. Proc. of Ninth IASTED International Conference on Power and Energy Systems PES 2007. Clearwater, Florida, USA, January 3-5, 2007. P. $128-133$.

17. Udut L.S., Chernyshev A.Yu., Gusev N.V. Development and simulation of scalar-controlled asynchronous electric drives. University News. Electromechanics, 2015, no. 6 (359), pp. 43-49. In Rus.

18. Herman I., Vaclavek P. Load torque and moment of inertia observability analysis for alternating current drive sensorless control. IECON Proceedings, Industrial Electronics Conference. Montreal, Canada, 2012. pp. 1864-1869.

19. Binder B.J.T., Pavlov A., Johansen T.A. Estimation of flow rate and viscosity in a well with an electric submersible pump using moving horizon estimation. IFAC-Papers on line, 2015, vol. 28 (6), pp. 140-146.

20. Rabbi S.F., Constantine M., Rahman M.A. A novel sensorless IPM motor drive for electric submersible pumps. IEEE International Electric Machines and Drives Conference. Miami, USA, 2017. Conference Paper № 8002199, pp. 1-8.

21. Messaoudi M., Sbita L. Sensorless direct torque and flux control of induction motor based on MRAS and Luenberger observer. International Conference on Green Energy and Conversion Systems. Hammamet, Tunisia, 2017. Conference Paper № 8066224, pp. 1-7.

22. Bahloul M., Chrifi-Alaoui L., Vargas A.N., Chaabane M., Drid S. Online robust estimation of flux and load torque in induction motors. International Journal of Advanced Manufacturing Techno$\log y, 2017$, vol. 94 (5-8), pp. 2703-2713.

Received: 12 September 2019.

\section{Information about the authors}

Tatyana A. Chernysheva, senior lecturer, National Research Tomsk Polytechnic University.

Vasiliy V. Anikin, senior lecturer, Nizhnevartovsk State University; postgraduate student, Omsk State Technical University.

Igor A. Chernyshev, Cand. Sc., associate professor, National Research Tomsk Polytechnic University.

Aleksandr Yu. Chernyshev, Cand. Sc., associate professor, National Research Tomsk Polytechnic University. 